\title{
Nuclear factor erythroid 2-related factor 2 antioxidant response element pathways protect bovine mammary epithelial cells against $\mathrm{H}_{2} \mathrm{O}_{2}$-induced oxidative damage in vitro
}

\author{
Y. F. Ma, ${ }^{* 1}$ Z. H. Wu, $\dagger^{1}$ M. Gao, ${ }^{* 2}$ and J. J. Loor $\ddagger^{2}$ \\ *Institute of Animal Nutrition and Feed, Inner Mongolia Academy of Agriculture and Animal Husbandry Sciences, Huhhot 010031, P. R. China \\ †College of Animal Science, Inner Mongolia Agricultural University, Hohhot 010018, P. R. China \\ ‡Department of Animal Sciences and Division of Nutritional Sciences, University of Illinois, Urbana 61801
}

\begin{abstract}
The experiment was conducted to determine the role of nuclear factor (erythroid-derived 2)-like factor 2 (NFE2L2, formerly Nrf2) antioxidant response element (ARE) pathway in protecting bovine mammary epithelial cells (BMEC) against $\mathrm{H}_{2} \mathrm{O}_{2}$-induced oxidative stress injury. An NFE2L2 small interfering RNA (siRNA) interference or a pCMV6-XL5-NFE2L2 plasmid fragment was transfected to independently downregulate or upregulate expression of NFE2L2. Isolated BMEC in triplicate were exposed to $\mathrm{H}_{2} \mathrm{O}_{2}(600$ $\mu M)$ for $6 \mathrm{~h}$ to induce oxidative stress before transient transfection with scrambled siRNA, NFE2L2-siRNA, pCMV6-XL5, and pCMV6-XL5-NFE2L2. Cell proliferation, apoptosis and necrosis rates, antioxidant enzyme activities, reactive oxygen species (ROS) and malondialdehyde (MDA) production, protein and mRNA expression of NFE2L2 and downstream target genes, and fluorescence activity of ARE were measured. The results revealed that compared with the control, BMEC transfected with NFE2L2-siRNA3 had proliferation rates that were 9 or $65 \%$ lower without or with $\mathrm{H}_{2} \mathrm{O}_{2}$, respectively. These cells also had apoptosis and necrosis rates that were 27 and 3.5 times greater with $\mathrm{H}_{2} \mathrm{O}_{2}$ compared with the control group, respectively. In contrast, transfected pCMV6-XL5-NFE2L2 had proliferation rates that were $64.3 \%$ greater or $17 \%$ lower without or with $\mathrm{H}_{2} \mathrm{O}_{2}$ compared with the control group, respectively. Apoptosis rates were 1.8 times lower with $\mathrm{H}_{2} \mathrm{O}_{2}$ compared with the control. In addition, compared with the control, production of ROS and MDA and activities of superoxide dismutase (SOD), glutathione peroxidase (GSH-Px), catalase (CAT), and glutathi-
\end{abstract}

Received November 12, 2017.

Accepted February 17, 2018.

${ }^{1}$ These authors contributed equally.

${ }^{2}$ Corresponding authors: gmyh1588@126.com and jloor@illinois.edu
one-S-transferase (GST) increased markedly in cells transfected with pCMV6-XL5-NFE2L2 and without $\mathrm{H}_{2} \mathrm{O}_{2}$. However, compared with the control, production of ROS and MDA and activity of CAT and GSH-Px increased markedly, whereas activities of SOD and GST decreased in cells transfected with pCMV6-XL5NFE2L2 and incubated with $\mathrm{H}_{2} \mathrm{O}_{2}$. Compared with the control, cells transfected with NFE2L2-siRNA3 with or without $\mathrm{H}_{2} \mathrm{O}_{2}$ had lower production of ROS and MDA and activity of SOD, CAT, GSH-Px, and GST. Cells transfected with pCMV6-XL5-NFE2L2 with or without $\mathrm{H}_{2} \mathrm{O}_{2}$ had markedly higher protein and mRNA expression of NFE2L2, heme oxygenase-1 (HMOX-1), NADH quinone oxidoreductase 1 , glutamate cysteine ligase catalytic subunit, and glutamyl cystine ligase modulatory subunit compared with the control incubations. Cells transfected with NFE2L2-siRNA3 without or with $\mathrm{H}_{2} \mathrm{O}_{2}$ had markedly lower protein and mRNA expression of NFE2L2, HMOX-1, NADH quinone oxidoreductase 1 , glutamyl cystine ligase modulatory subunit, and glutamate-cysteine ligase catalytic subunit compared with the control incubations. In addition, expression of HMOX-1 was 5.3-fold greater with $\mathrm{H}_{2} \mathrm{O}_{2}$ compared with the control. Overall, results indicate that NFE2L2 plays an important role in the NFE2L2-ARE pathway via the control of HMOX-1. The relevant mechanisms in vivo merit further study.

Key words: lactation, mammary gland, oxidative stress, antioxidant

\section{INTRODUCTION}

The periparturient period is the most critical period during the lactation cycle of dairy cattle (Loor et al., 2013), in large part because dairy cattle are most susceptible to infectious diseases during this time (Goff, 2006). A major contributing factor for the increased incidence of health disorders is the dysfunction of immune and oxidative stress (OS) responses (Sordillo 
et al., 2009; Sordillo and Aitken, 2009; Deng et al., 2017). In addition, the proliferation and apoptosis of bovine mammary epithelial cells (BMEC) under OS are modulated by cellular antioxidant status (Miranda et al., 2011).

Aside from applied research targeted at milk production, lactation, and mammary carcinogenesis, the mammary gland is an ideal experimental model system for resolving fundamental questions in areas extending from cellular and developmental biology to endocrinology and biotechnology (Ahn et al., 1995). In vitro culture systems have become increasingly important in modern research because they offer the potential to manipulate the growth and differentiation of cells through simulating the endocrine in vivo environment (Howlett and Bissell, 1993).

Apoptosis and proliferation rate control the death and survival of BMEC (Zarzyńska et al., 2007), and previous research revealed that OS can increase the rate of apoptosis leading to cell and tissue necrosis (Fernández-Checa, 2003). Although mammary cell proliferation rate during established lactation is low, it is not insignificant in relation to maintaining cell number (Fernández-Checa, 2003).

An imbalance between excessive formation of reactive oxygen species (ROS) and reactive nitrogen species, the reduced antioxidant capacity of cells, or both result in OS (Kruk and Duchnik, 2014). During normal cellular metabolism, the mitochondrion produces ROS including oxygen ions, free radicals, and lipid hydroperoxides as byproducts of the electron transport chain reaction (Szőcs, 2004; Sordillo and Aitken, 2009). However, in the absence of antioxidant mechanisms to counteract the excessive production of these molecules, the onset of OS can directly increase damage of proteins and DNA and induce lipid peroxide production [e.g., malondialdehyde (MDA); Li et al., 2016], all of which can overwhelm tissue-repair mechanisms and eventually cause irreversible cell and tissue damage (Bouwstra et al., 2010). It is now recognized that ROS generated through a variety of extracellular and intracellular actions act as signaling mediators that can affect growth, differentiation, progression, and cell death (Zhang et al., 2013; Deng et al., 2017).

The Kelch-like epichlorohydrin-associated protein 1 (Keap1)-nuclear factor erythroid 2-related factor 2 (NFE2L2)-antioxidant response element (ARE) signaling pathway performs critical roles in maintaining cellular redox balance and metabolism. It can induce an adaptive response against OS that can otherwise lead to uncontrolled inflammation. Under higher rates of oxidative metabolism, such as after parturition, an increase of intracellular ROS can promote the dissocia- tion of Keap1-NFE2L2-ARE (Nguyen et al., 2003; Kaspar et al., 2009; Kim et al., 2010), after which NFE2L2 transfers to the nucleus and binds to ARE on target genes, inducing a cascade of events designed to prevent OS (Jain and Jaiswal, 2007; Kensler et al., 2007). Some evidence indicates that the Keap1-NFE2L2-ARE pathway, which is functional in BMEC (Jin et al., 2016a,b), may be the most important mechanism in ruminants for protecting cells from OS (Kaspar et al., 2009; Jin et al., 2016b).

One of the few studies with BMEC recently revealed that the Keap1-NFE2L2-ARE antioxidant pathway and its downstream antioxidant enzyme heme oxygenase-1 (HMOX-1) have a crucial role in the ability of BMEC to cope with $\mathrm{H}_{2} \mathrm{O}_{2}$-induced OS (Jin et al., 2016a). Phase II detoxifying enzymes provide a major mechanism by which cells combat the toxicity of electrophiles and ROS, and their induction is highly effective and sufficient for protecting cells against toxic challenges (Talalay, 2000). These enzymes include HMOX-1, NADH quinone oxidoreductase 1 (NQO1), glutamatecysteine ligase catalytic subunit (GCLC), and glutamyl cystine ligase modulatory subunit (GCLM). The antioxidant enzymes superoxide dismutase (SOD), catalase (CAT), glutathione-S-transferase (GST), and glutathione peroxidase (GSH-Px) are controlled by activated Keap1-NFE2L2-ARE (Kaspar et al., 2009).

Because available data indicate that BMEC might be susceptible to OS and because the Keap1-NFE2L2ARE pathway is an important mechanism in the cellular defense against OS, we hypothesized that dysfunction in the Keap1-NFE2L2-ARE signaling pathway may result in increased sensitivity to $\mathrm{H}_{2} \mathrm{O}_{2}$-induced OS. If this hypothesis is proven correct, it could help develop means to target the pathway in vivo, specifically during the periparturient period, when cows are most susceptible to OS and mastitis. The present study sought to downregulate and upregulate NFE2L2 via silencing using small interfering RNA (siRNA) or transfecting an NFE2L2 construct to elucidate the effects of $\mathrm{H}_{2} \mathrm{O}_{2}$ on the Keap1-NFE2L2-ARE pathway in BMEC.

\section{MATERIALS AND METHODS}

\section{Cell Culture and Treatment}

Mammary tissue was harvested from five 4-yr-old late-lactation dairy cows from a local slaughterhouse (Hohhot, China). The midpoint area of the left rear side of the udder was clipped and surgically scrubbed. Approximately $150 \mathrm{mg}$ of fresh tissue from each cow was removed and placed in sterilized tubes containing ice-cold Dulbecco's PBS (Sigma-Aldrich, St. Louis, 
MO) and immediately transported to the laboratory. The samples were washed with Dulbecco's PBS 3 times and cut into $1-\mathrm{mm}^{3}$ pieces. Tissue was transferred with tweezers onto clean, sterile plastic cell culture dishes (Corning Inc., Corning, NY). The culture dishes were maintained in a humidified incubator at $37^{\circ} \mathrm{C}$ in an atmosphere of $5 \% \mathrm{CO}_{2}$ and $95 \%$ air. After $4 \mathrm{~h}, 5 \mathrm{~mL}$ of basal medium was added into the culture. The basal medium was replaced with fresh medium every 24 or 48 $\mathrm{h}$ until the cells were distributed across the bottom of the dish. Subsequently, epithelial cells were enriched by selective detachment with trypsinization using $0.25 \%$ trypsin (Gibco, Grand Island, NY). After 3 min of trypsinization, fibroblasts were removed by washing with Dulbecco's PBS. The epithelial cells attached to the dish surface were allowed to grow by addition of fresh medium. The BMEC were continuously purified using the same method. The purified BMEC were seeded at a density of $5 \times 10^{5}$ cells in culture flasks and continuously subcultured up to 60 passages. For cryopreservation, $1 \times 10^{6}$ cells $/ \mathrm{mL}$ was suspended in freezing medium. A supraphysiologic concentration of $600 \mu M \mathrm{H}_{2} \mathrm{O}_{2}$ was applied to the BMEC to induce OS. In a preliminary unpublished study including doses of 0 to $1,000 \mu M \mathrm{H}_{2} \mathrm{O}_{2}$, we used data on cell proliferation, concentration of ROS, and activity of SOD and GSH-Px to determine an optimal dose for the study described in this paper. Basal medium comprised 85.74 $\mathrm{mL}$ of Dulbecco's modified Eagle medium (DMEM; 12400-024, Gibco) and $10 \mathrm{~mL}$ of $10 \%$ (vol/vol) fetal bovine serum (Gibco).

\section{Transient Transfection of NFE2L2}

Small interference RNA (Beyotime Institute of Biotechnology, Shanghai, China) was used to silence the NFE2L2 gene. In brief, BMEC $\left(2 \times 10^{6}\right.$ cells/well for 6 -well plates and $2 \times 10^{5}$ cells/well for 96 -well plates) were plated into each well and cultured for $24 \mathrm{~h}$. For overexpression and interference of NFE2L2, cells were transfected with pCMV6-XL5-NFE2L2 and NFE2L2siRNA3 at 90 to $95 \%$ confluence using Lipofectamine 2000 transfection reagent (Gibco). After $12 \mathrm{~h}$ of transfection, cells were recovered in basal medium after removal of transfection reagents and were then incubated for an additional $36 \mathrm{~h}$ without (control) or with (OS) $\mathrm{H}_{2} \mathrm{O}_{2}$ treatment. The BMEC in 6-well plates were harvested $48 \mathrm{~h}$ after transfection for assessing mRNA and protein expression. For the cells cultured in 96-well plates, the culture medium was switched to serum-free, antibiotic-free DMEM $48 \mathrm{~h}$ after transfection for assessing cell proliferation rate, and cells were then subjected to $600 \mu M \mathrm{H}_{2} \mathrm{O}_{2}$ for $6 \mathrm{~h}$. The BMEC proliferation rate of control, pCMV6-XL5 transfected and scrambled siRNA transfected (minus $\mathrm{H}_{2} \mathrm{O}_{2}$ ), pCMV6-XL5-NFE2L2 transfected and NFE2L2-siRNA3 transfected (minus $\mathrm{H}_{2} \mathrm{O}_{2}$ ), pCMV6-XL5 transfected and scrambled siRNA transfected (plus $600 \mu M \mathrm{H}_{2} \mathrm{O}_{2}$ ), and pCMV6-XL5-NFE2L2 transfected and NFE2L2-siRNA3 transfected (600 $\mu M$ $\mathrm{H}_{2} \mathrm{O}_{2}$ ) was then measured.

\section{RNA Interference}

Three siRNA specific to NFE2L2 (NFE2L2-siRNA1, NFE2L2-siRNA2, and NFE2L2-siRNA3) and a scrambled sequence (scrambled siRNA) were designed and synthesized by Shanghai Genechem Co. Ltd. (Shanghai, China). The siRNA sequences are listed in Table 1. The BMEC were transfected in antibiotic-free medium following the manufacturer's instructions (Shanghai Genechem Co. Ltd.). Briefly, healthy BMEC were routinely trypsinized and resuspended to $2 \times 10^{6}$ cells/ $\mathrm{mL}$ in normal growth medium without antibiotics, and cells were then set aside at $37^{\circ} \mathrm{C}$. After $24 \mathrm{~h}$, the cells were transfected with NFE2L2-siRNA. After incubation for $5 \mathrm{~min}$ at $37^{\circ} \mathrm{C}$, the siRNA and Lipofectamine were mixed, incubated at room temperature for an additional $20 \mathrm{~min}$, and then added to each well. The cell suspension that was previously set aside was transferred to the same wells containing the complex $(2 \times$ $10^{6}$ cells per well for 6 -well plates and $2 \times 10^{5}$ cells per well for 96-well plates), and the cell suspension was mixed by gently rocking the plates back and forth. The basal medium was replaced by the same fresh medium $12 \mathrm{~h}$ after transfection. The BMEC in 6-well plates were harvested $48 \mathrm{~h}$ after transfection to assess mRNA and protein changes. At $48 \mathrm{~h}$ after transfection, the culture medium for the BMEC in 96-well plates was switched to serum-free, antibiotic-free DMEM, and the cells were then subjected to $600 \mu M \mathrm{H}_{2} \mathrm{O}_{2}$ for $6 \mathrm{~h}$. The cell proliferation rate of NFE2L2-silenced and control culture was measured. Scrambled transfections (using a

Table 1. Primer sequences for the nuclear factor erythroid 2-related factor 2 (NFE2L2) small interfering RNA (siRNA) tested

\begin{tabular}{ll}
\hline Primer & Primer sequence $\left(5^{\prime} \rightarrow 3^{\prime}\right.$ orientation $)$ \\
\hline NFE2L2-siRNA1 & TTGCTCAAAGAAAGAGGAGAA \\
Sense & TTCTCCTCTTTCTTTGAGCAA \\
Antisense & ACTGGATAAAGAAGTAGGTAA \\
NFE2L2-siRNA2 & TTACCTACTTCTTTATCCAGT \\
Sense & \\
Antisense & CTGGAGCAAGATTTAGATCAT \\
NFE2L2-siRNA3 & ATGATCTAAATCTTGCTCCAG \\
Sense & \\
\hline
\end{tabular}


nontarget siRNA) were performed in each experiment. Three different NFE2L2-specic siRNA were tested.

\section{BMEC Proliferation Rate Assay}

To quantify BMEC proliferation rate, BMEC were plated at a density of $2 \times 10^{6}$ cells per well for 96-well plates overnight, and the cells were then transfected with scrambled siRNA, NFE2L2-siRNA3, pCMV6-XL5, and pCMV6-XL5-NFE2L2. At $48 \mathrm{~h}$ after transfection, the culture medium was switched to serum-free, antibioticfree DMEM, and the cells were then exposed to 600 $\mu M \mathrm{H}_{2} \mathrm{O}_{2}$ for $6 \mathrm{~h}$. The BMEC proliferation rate was performed using the 3-(4, 5-dimethylthiazol-2-yl)-5-(3carboxymethoxyphenyl)-2-(4-sulfophenyl)-2H-tetrazolium (MTS) assay kit (G3582, Promega, Madison, WI) according to the manufacturer's instructions. Briefly, $20 \mu \mathrm{L}$ of MTS was added to each well and incubated for $4 \mathrm{~h}$ at $37^{\circ} \mathrm{C}$. Subsequently, $150 \mu \mathrm{L}$ of dimethyl sulfoxide (Sigma-Aldrich) was added to each well and incubated for $10 \mathrm{~min}$ at $37^{\circ} \mathrm{C}$. Last, the absorbance at $490 \mathrm{~nm}$ was determined with a microplate reader (Molecular Devices, Sunnyvale, CA).

\section{Detection of OS Indicators}

The activities of SOD, CAT, GSH-Px, and GST as well as the contents of MDA in BMEC were determined by using spectrophotometric diagnostic kits from Nanjing Jiancheng Biotechnology Institute (Nanjing, China) according to the manufacturer's protocols. In brief, BMEC were washed with PBS and incubated with fresh DMEM (Gibco) at $37^{\circ} \mathrm{C}$ for $35 \mathrm{~min}$, and then $1 \times 10^{6}$ cells were harvested and resuspended in PBS. Absorbance was detected at $560 \mathrm{~nm}$ (SOD), $420 \mathrm{~nm}$ (GSH-Px), $405 \mathrm{~nm}$ (CAT), $450 \mathrm{~nm}$ (GST), and $532 \mathrm{~nm}$ (MDA) and was recorded with a $722 \mathrm{~N}$ spectrophotometer (Scientific Instrument Co. Ltd., Shanghai, China).

\section{Intracellular ROS Detection}

A dichlorofluorescein staining assay was used to detect intracellular ROS. Briefly, BMEC were washed with PBS and incubated with fresh DMEM containing $10 \mu \mathrm{M}$ dichlorofluorescein at $37^{\circ} \mathrm{C}$ for $35 \mathrm{~min}$, and then $1 \times 10^{6}$ cells were harvested and resuspended in PBS. The optical density at $450 \mathrm{~nm}$ was recorded with a microplate reader (Molecular Devices).

\section{ARE Fluorescence Activity}

The ARE fluorescence activity was tested in cultured BMEC with or without $\mathrm{H}_{2} \mathrm{O}_{2}$ via an ARE reporter gene according to the manufacturer's instructions (Promega). In brief, after the treatment of BMEC with $\mathrm{H}_{2} \mathrm{O}_{2}(600$ $\mu M$ ) for $0,6,12$, and $24 \mathrm{~h}, 50 \mu \mathrm{L}$ of luciferase assay reagent II was added to the BMEC, followed by $10 \mu \mathrm{L}$ of cell lysis buffer. The activity of relative light unit 1 (RLU1) was detected with a biluciferase reporter gene kit (E1910; Promega). Then, $50 \mu \mathrm{L}$ of $50 \times$ Stop and Glo reagent was added to the BMEC, and the activity of relative light unit 2 (RLU2) was detected again with a biluciferase reporter gene kit (E1910; Promega). The RLU1/RLU2 ratio was used to determine the transfection efficiency of ARE.

\section{RNA Isolation, cDNA Synthesis, and Quantitative PCR}

Total RNA was isolated from BMEC using the miRNeasy kit (Qiagen, Hilden, Germany) following the manufacturer's protocols. Samples were treated on-column with DNaseI (Qiagen, Hilden, Germany); quantification was assessed using the NanoDrop ND-1000 (NanoDrop Technologies, Wilmington, DE), and RNA quality was measured using an Agilent 2100 Bioanalyzer (Agilent, Santa Clara, CA). All samples had an RNA integrity number factor greater than 6.3. The quantitative PCR was performed as described previously (Osorio et al., $2014)$ to determine the relative mRNA abundance of NFE2L2, HMOX1, NQO1, GCLC, GCLM, and TUBB (tubulin $\beta$; internal control). All reactions were run in triplicate. The NFE2L2-siRNA1, 2, and 3 and primer sequences are presented in Table 1 . The primers of NFE2L2 and downstream target genes used for reversetranscription PCR are listed in Table 2.

Table 2. Primers of downstream target genes in the nuclear factor erythroid 2-related factor 2 (NFE2L2) antioxidant response element (ARE) signaling pathway

\begin{tabular}{|c|c|c|c|}
\hline Gene name & Forward primer $\left(5^{\prime} \rightarrow 3^{\prime}\right)$ & Reverse primer $\left(5^{\prime} \rightarrow 3^{\prime}\right)$ & Accession no. \\
\hline NFE2L2 & CCAGCACAACACATACCA & TAGCCGAAGAAACCTCATT & 001011678.2 \\
\hline$H M O X 1$ & GAACGCAACAAGGAGAAC & CTGGAGTCGCTGAACATAG & 001014912.1 \\
\hline NQO1 & CAACAGACCAGCCAATCA & ACCTCCCATCCTTTCCTC & 001034535.1 \\
\hline$G C L C$ & ATTGGGTGGAGAGTGGAA & ACAGCGGGATGAGAAAGT & 001083674.1 \\
\hline$G C L M$ & CCGATGAAAGAGAAGAAATG & CAACAGGAGGTGAAGCAA & 001038143.1 \\
\hline
\end{tabular}




\section{Western Blot Analysis}

After treatment, cells were washed twice with $4^{\circ} \mathrm{C}$ PBS (0.01 $M, \mathrm{pH} 7.2-7.3)$ and were then lysed with radio-immunoprecipitation assay protein lysate (Beyotime Institute of Biotechnology, Shanghai, China). Total protein was extracted from BMEC using a tissue protein extraction reagent (cat. no. 78510; Thermo Scientific, Waltham, MA) containing inhibitor cocktail $(100 \times$, cat. no. 78442 ; Thermo Scientific). Total protein concentration was determined using the BCA protein assay kit (Sigma-Aldrich). Protein samples were boiled for $20 \mathrm{~min}$ at $100^{\circ} \mathrm{C}$; $50 \mathrm{~g}$ of total protein per lane was resolved by $10 \%$ SDS-PAGE (Bio-Rad, Hercules, $\mathrm{CA}$ ) and then transferred from the gel to a polyvinylidene difluoride membrane $(0.45 \mu \mathrm{m}$; Millipore, Billerica, MA). PageRuler Plus prestained protein ladder (Fermentas, Hanover, MD) was used to confirm protein electrophoresis. Membranes were blocked in Tris-buffered saline with Tween (TBST; $50 \mathrm{~m} M$ Tris, $\mathrm{pH} 7.6 ; 150 \mathrm{mM} \mathrm{NaCl}$; and $0.1 \%$ Tween 20 ) that contained $5 \%$ (wt/vol) nonfat dry milk for $2 \mathrm{~h}$ at room temperature with gentle agitation. The membranes were then incubated in TBST containing antibodies to NFE2L2 (cat. no. ab137550; Abcam, Cambridge, MA), HMOX-1 (cat. no. ab13248; Abcam), NQO1 (cat. no. ab28947; Abcam), GCLC (cat. no. ab80841; Abcam), and GCLM (cat. no. ab81445, Abcam) with gentle agitation at $4^{\circ} \mathrm{C}$ overnight. After incubating with primary antibody, the membranes were washed and incubated with horseradish peroxidase (HRP)-conjugated secondary antibodies (HRP labeled sheep anti-mouse, 1:50,000, and HRP labeled sheep anti-rabbit, 1:50,000; cat. no. ab6721; Abcam) in TBST for $1 \mathrm{~h}$ at room temperature. The membranes were washed and then incubated with electro-chemi-luminescence reagent (cat. no. 170-5060; Bio-Rad). Tubulin (cat. no. ab56676; Abcam) and $\beta$-actin (cat. no. ab8226; Abcam) were used as internal controls. The images were captured using Chemi DOC MP (Bio-Rad). The intensities of the bands were measured with Image-Pro Plus 6.0 software (Media Cybernetics Inc., Rockville, MD).

\section{BMEC Apoptosis and Necrosis Rate}

To quantify apoptotic and necrotic death, BMEC were plated at a density of $2 \times 10^{6}$ cells per well for 96 well plates overnight, and the cells were then transfected with scrambled siRNA, NFE2L2-siRNA3, pCMV6-XL5, and pCMV6-XL5-NFE2L2. At $48 \mathrm{~h}$ after transfection, the culture medium was switched to serum-free, antibiotic-free DMEM, and the cells were then exposed to
$600 \mu M \mathrm{H}_{2} \mathrm{O}_{2}$ for $6 \mathrm{~h}$. The apoptosis rate and necrosis rate of cells were detected with the dead cell apoptosis kit (V13241, Thermo Fisher). Briefly, $5 \mu \mathrm{L}$ of Alexa Fluor 488 Annexin V (V13241, Thermo Fisher) and 1 $\mu \mathrm{L}$ of $100 \mu \mathrm{g} / \mathrm{mL}$ propidium iodide working solution was added to each $100 \mu \mathrm{L}$ of cell suspension, and the cells were incubated at room temperature for $15 \mathrm{~min}$. Subsequently, $400 \mu \mathrm{L}$ of $1 \times$ annexin-binding buffer was added to each well and mixed gently, and the samples were kept on ice. The stained cells were subsequently analyzed by flow cytometry (FACSCantoII, BD Biosciences, San Jose, CA), and fluorescence emission was measured at $530 \mathrm{~nm}$.

\section{Statistical Analysis}

All statistical tests were carried out using SAS 9.0 (SAS Institute Inc., Cary, NC). One-way ANOVA followed by a Student-Newman-Keuls multiple-comparison test was used to determine statistical significance for multiple comparisons, and a Student's t-test was used for 2 group comparisons. All data are expressed as means \pm standard error of the mean for the indicated number of independent experiments performed. Differences were considered significant at $P<0.05$.

\section{RESULTS}

\section{Verification and Screening of NFE2L2-siRNA Interference Fragment}

Compared with the control group, the BMEC transfected with NFE2L2-siRNA1, NFE2L2-siRNA2, and NFE2L2-siRNA3 had reduced protein and mRNA expression of NFE2L2; the best interference efficiency was achieved with NFE2L2-siRNA3 $(>50 \% ; P<0.01$; Figure 1). Therefore, NFE2L2-siRNA3 was chosen as the interference fragment for subsequent experiments.

\section{Effect of Downregulation and Upregulation of NFE2L2 on the Proliferation Rate of BMEC}

The transfection with NFE2L2-siRNA3 decreased the proliferation rate by $65 \%(P<0.05)$ under OS compared with the control group (Figure 2). This result suggested that downregulation of NFE2L2 decreased the ability of BMEC to cope with $\mathrm{H}_{2} \mathrm{O}_{2}$-induced cytotoxicity. Transfection with pCMV6-XL5 and pCMV6XL5-NFE2L2 without $\mathrm{H}_{2} \mathrm{O}_{2}$ increased the proliferation rate by $50 \%(P<0.01)$ and $180 \%(P<0.01)$, respectively, compared with the control group (Figure 2). 
A

NFE2L 2

Tubulin
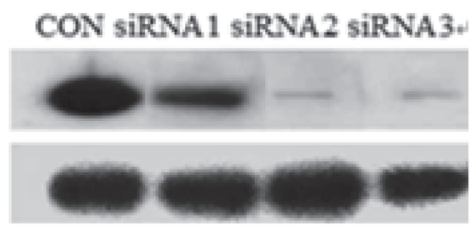

B

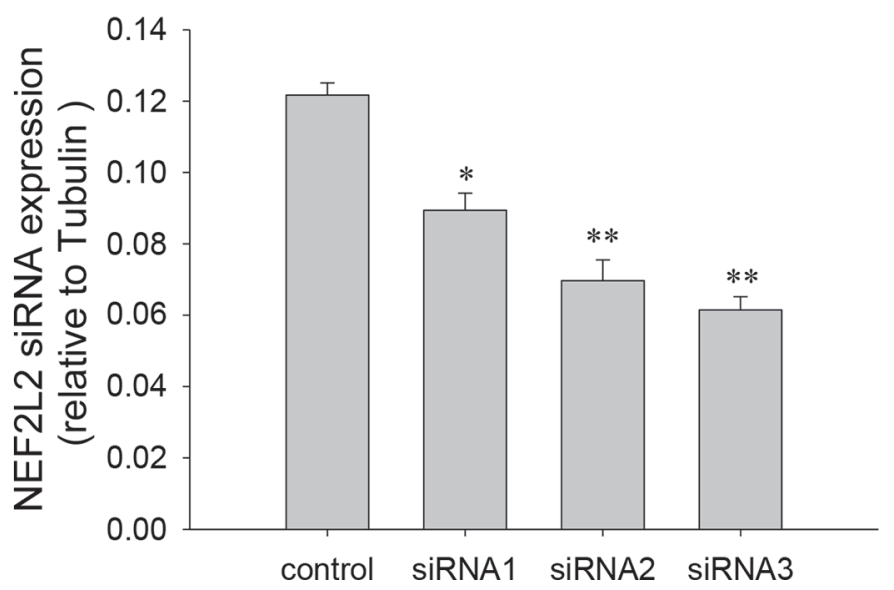

Figure 1. Screening for efficacy of nuclear factor erythroid 2-related factor 2 (NFE2L2) small interfering RNA (siRNA) fragments. The mRNA and protein expression of NFE2L2 after siRNA treatment was detected by reverse-transcription PCR and Western blot. ${ }^{*} P<$ $0.05,{ }^{* *} P<0.01$ compared with the control group. Error bars indicate SEM.

\section{Effect of Downregulation and Upregulation of NFE2L2 on Apoptosis and Necrosis Rate in BMEC Under OS}

The results revealed that transfection with NFE2L2siRNA3 with $\mathrm{H}_{2} \mathrm{O}_{2}$ sharply increased $(P<0.01)$ the apoptosis and necrosis rate by 4.5 - and 5 -fold, respectively, compared with the control group. However, transfection with pCMV6-XL5-NFE2L2 plus $\mathrm{H}_{2} \mathrm{O}_{2}$ sharply decreased $(P<0.05)$ the apoptosis rate by $70 \%$ compared with the control group (Figure 3). These results suggested that downregulation of NFE2L2 sharply decreased the ability of BMEC to cope with $\mathrm{H}_{2} \mathrm{O}_{2^{-}}$ induced apoptosis, whereas upregulation of NFE2L2 sharply enhanced the ability of BMEC to cope with $\mathrm{H}_{2} \mathrm{O}_{2}$-induced apoptosis.

\section{Effect of Downregulation and Upregulation of NFE2L2 on Antioxidant Enzyme Activities in BMEC}

Transfection of NFE2L2-siRNA3 without $\mathrm{H}_{2} \mathrm{O}_{2}$ sharply decreased SOD $(P<0.05)$, CAT $(P<0.01)$, GSH-Px $(P<0.01)$, and GST $(P<0.01)$ activity and
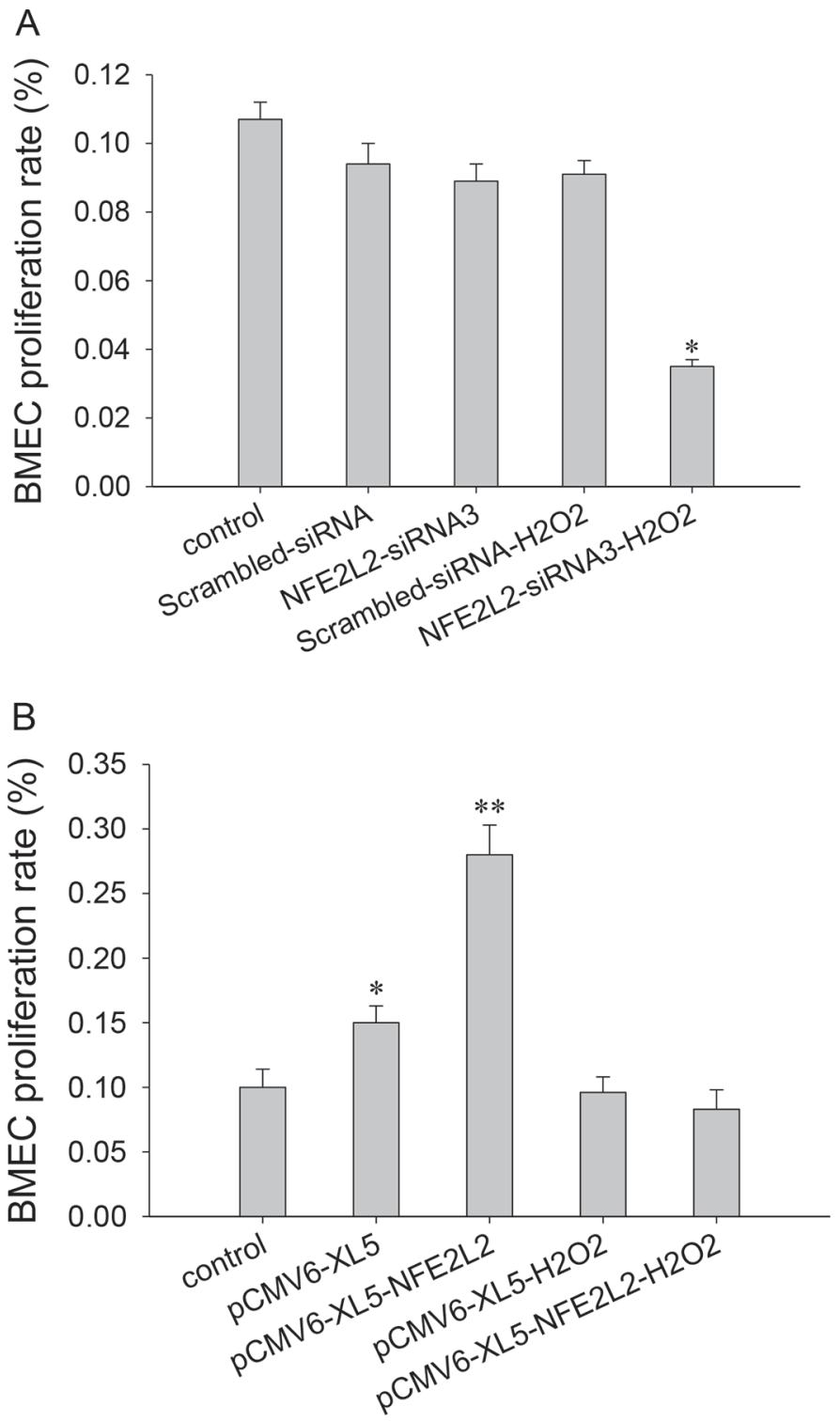

Figure 2. Effect of regulating nuclear factor erythroid 2-related factor 2 (NFE2L2) expression on bovine mammary epithelial cell (BMEC) proliferation rate. (A) The BMEC were transiently transfected with control fragment scrambled small interfering RNA (siRNA) and the interference fragment NFE2L2 siRNA3 for $48 \mathrm{~h}$ under normal conditions, followed by transfection with scrambled siRNA and NFE2L2 siRNA3 for $48 \mathrm{~h}$ after treatment with $\mathrm{H}_{2} \mathrm{O}_{2}(600 \mu M)$ for $6 \mathrm{~h}$. (B) The BMEC were transiently transfected with the empty plasmid PCMV6-XL5 and the overexpression vector pCMV6-XL5 NFE2L2 for $48 \mathrm{~h}$ under normal conditions followed by transfection with PCMV6XL5 and pCMV6-XL5 NFE2L2 for $48 \mathrm{~h}$ after treatment with $\mathrm{H}_{2} \mathrm{O}_{2}$ $(600 \mu M)$ for 6 h. ${ }^{*} P<0.05,{ }^{* *} P<0.01$ compared with the control group. Error bars indicate SEM.

ROS $(P<0.01)$ and MDA $(P<0.05)$ production compared with the control group, respectively (Figure 4 ). However, transfecting with NFE2L2-siRNA3 plus $\mathrm{H}_{2} \mathrm{O}_{2}$ sharply decreased SOD $(P<0.01)$, CAT $(P<0.001)$, GSH-Px $(P<0.001)$, and GST $(P<0.001)$ activity as 
well as concentration of ROS $(P<0.01)$ and MDA $(P$ $<0.01)$ compared with the control group.

The transfection of pCMV6-XL5-NFE2L2 without $\mathrm{H}_{2} \mathrm{O}_{2}$ sharply increased SOD $(P<0.05)$, CAT $(P<$ $0.001)$, and GSH-Px $(P<0.01)$ activity compared with the control group (Figure 5). However, transfection with pCMV6-XL5-NFE2L2 plus $\mathrm{H}_{2} \mathrm{O}_{2}$ increased CAT $(P<0.01)$ activity as well as $\operatorname{ROS}(P<0.05)$ and MDA $(P<0.05)$ production and sharply decreased SOD $(P<0.01)$ and GST $(P<0.01)$ activity compared with the control group. These results further support the hypothesis that NFE2L2 is essential for induction of several antioxidant defense enzymes in BMEC under OS. Results also suggest that the antioxidant enzyme system is impaired in BMEC exposed to supraphysiologic levels of $\mathrm{H}_{2} \mathrm{O}_{2}$.

\section{Effect of Regulating NFE2L2 Expression on Target Genes in the NFE2L2-ARE Pathway}

Figures 6 and 7 depict the expression of NFE2L2 and 4 main phase II detoxifying genes in BMEC after treatment with or without $600 \mu M \mathrm{H}_{2} \mathrm{O}_{2}$ for $6 \mathrm{~h}$. The western blot and reverse-transcription PCR results demonstrated that transfection of NFE2L2-siRNA3 without $\mathrm{H}_{2} \mathrm{O}_{2}$ sharply decreased the protein and mRNA expression of NFE2L2 $(P<0.05)$, HMOX-1 $(P<0.01)$, NQO1 $(P<0.05)$, GCLM $(P<0.05)$, and GCLC $(P$ $<0.05)$ compared with the control group. Furthermore, those results were lower compared with the transfec-

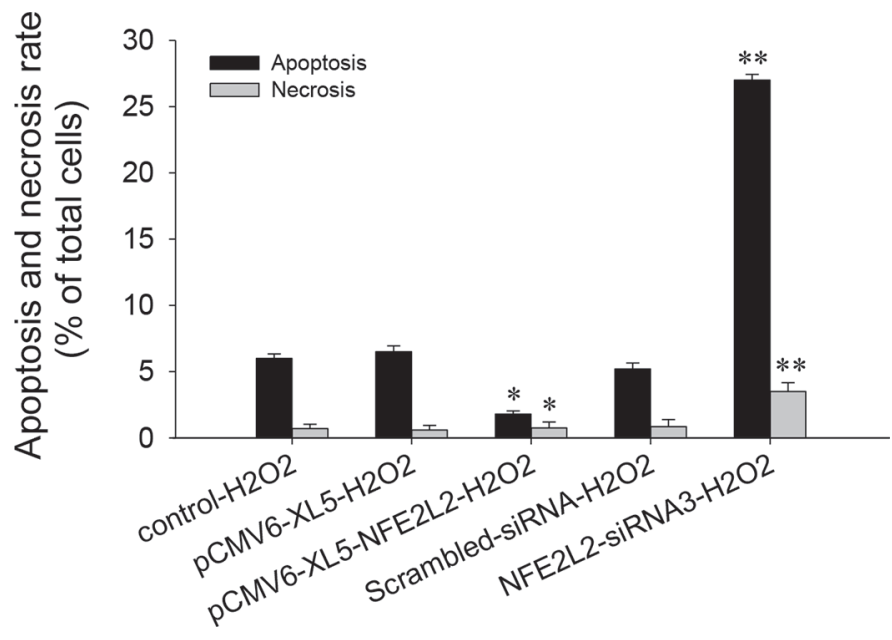

Figure 3. Effect of regulating nuclear factor erythroid 2-related factor 2 (NFE2L2) expression on apoptosis and necrosis rate of bovine mammary epithelial cells (BMEC) under oxidative stress. Apoptosis and necrosis rate of BMEC were detected following treatment with $\mathrm{H}_{2} \mathrm{O}_{2}(600 \mu M)$ for $6 \mathrm{~h}$ followed by transfection with pCMV6-XL5 and PCMV6-XL5 NFE2L2, scrambled small interfering RNA (siRNA), and NFE2L2 siRNA3 for 48 h. ${ }^{*} P<0.05,{ }^{*} P<0.01$ compared with the control group. Error bars indicate SEM. tion of scrambled siRNA $(P<0.05)$. The transfection of NFE2L2-siRNA3 with $\mathrm{H}_{2} \mathrm{O}_{2}$ sharply decreased the protein and mRNA expression of NFE2L2 $(P<0.01)$, HMOX-1 $(P<0.05)$, NQO1 $(P<0.01)$, GCLM $(P<$ $0.05)$, and GCLC $(P<0.05)$ compared with the control group. Furthermore, those results were significantly lower compared with the transfection of scrambled siRNA $(P<0.05)$. Overall, these results suggested that downregulation of NFE2L2 decreased the expression of NFE2L2 and all 4 genes regardless of $\mathrm{H}_{2} \mathrm{O}_{2}$.

The transfection of pCMV6-XL5-NFE2L2 without $\mathrm{H}_{2} \mathrm{O}_{2}$ sharply increased the protein and mRNA expression of HMXO-1 $(P<0.05)$, NQO1 $(P<0.05)$, and GCLM $(P<0.05)$ compared with the control group, respectively, and the response was significantly higher compared with the transfection of pCMV6-XL5 ( $P$ $<0.05$; Figures 8 and 9). However, transfection with pCMV6-XL5-NFE2L2 plus $\mathrm{H}_{2} \mathrm{O}_{2}$ increased the protein and mRNA expression of NFE2L2 $(P<0.01)$, NQO1 $(P<0.05)$, GCLM $(P<0.01)$, and GCLC $(P<0.05)$ compared with the control group, respectively. Overall, these results suggested that upregulation of NFE2L2 enhanced the expression levels of NFE2L2 and all 4 genes regardless of $\mathrm{H}_{2} \mathrm{O}_{2}$. The most significant change was detected for HMOX-1, with a 5.3-fold increase when compared with the control.

\section{HMOX-1 Is a Main Target Gene of the NFE2L2-ARE Pathway}

Incubation with $\mathrm{H}_{2} \mathrm{O}_{2}$ led to a greater than 5.3-fold increase in $H M O X-1$ mRNA expression in oxidativedamaged BMEC, whereas the increases in NQO1, $G C L M$, and $G C L C$ mRNA expression were of smaller magnitude, with only a 2 - to 2.8 -fold increase compared with the $\mathrm{H}_{2} \mathrm{O}_{2}$-untreated group (control group; Figure $10)$.

\section{Impaired NFE2L2 Transcriptional Activity in the NFE2L2-ARE Pathway}

To compare the NFE2L2-ARE transcriptional activity between control and oxidative damage, we used a dual-luciferase reporter assay to measure ARE luciferase activity in the presence and absence of $\mathrm{H}_{2} \mathrm{O}_{2}$. The results revealed that treatment with $\mathrm{H}_{2} \mathrm{O}_{2}$ increased ( $P$ $<0.05)$ the transcriptional activity of NFE2L2 in terms of ARE luciferase activity in a time-dependent manner in both control and oxidative-damaged groups. In contrast, ARE luciferase activity of oxidative-damaged BMEC was much lower $(P<0.05)$ than control BMEC (Figure 11). These data clearly indicate that NFE2L2driven transcriptional activation of ARE is aberrant in oxidative-damaged BMEC. 

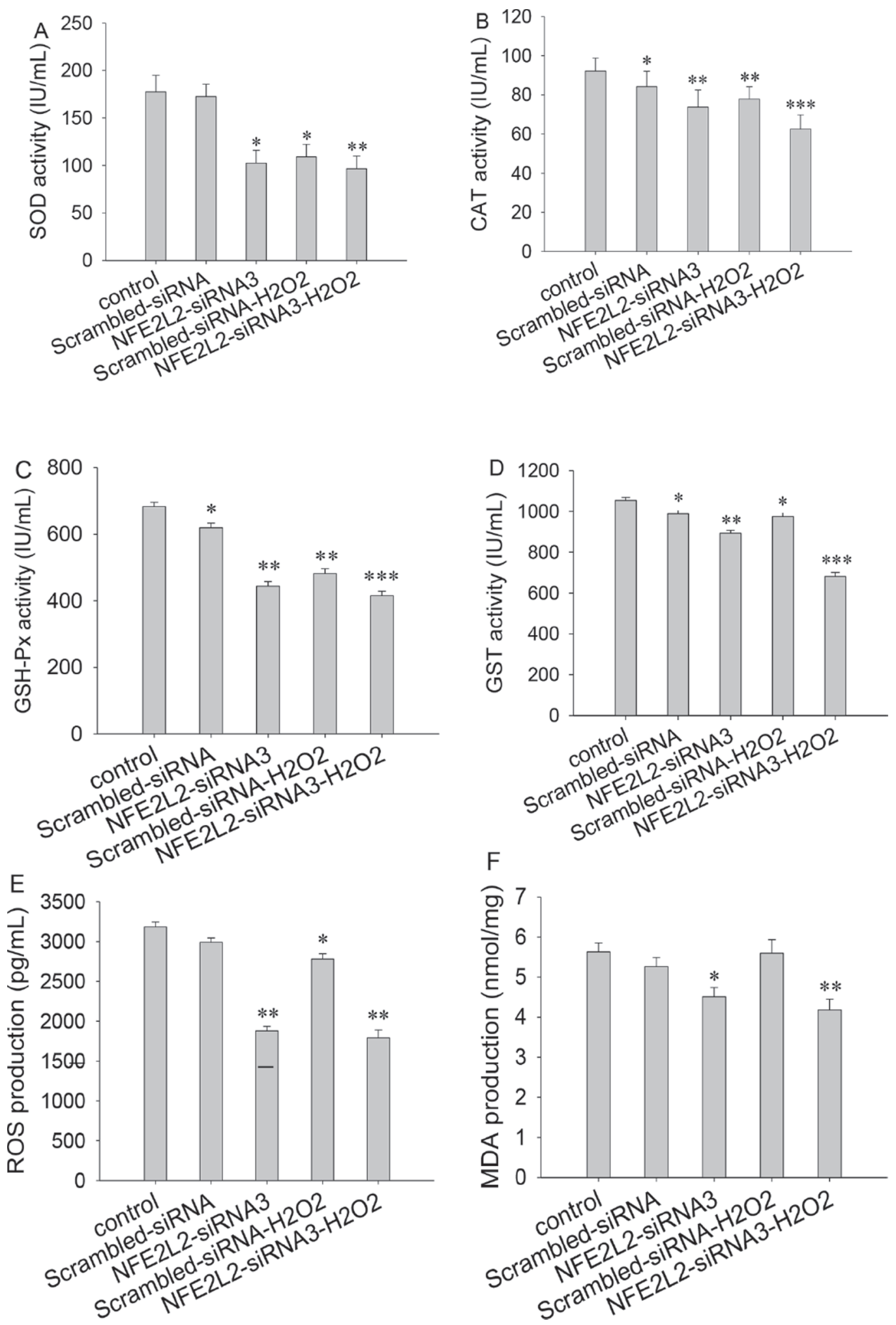

Figure 4. Effect of downregulating nuclear factor erythroid 2-related factor 2 (NFE2L2) expression on antioxidative enzyme activity, reactive oxygen species (ROS), and malondialdehyde (MDA) production in bovine mammary epithelial cells (BMEC). Activity of (A) superoxide dismutase (SOD), (B) catalase (CAT), (C) glutathione peroxidase (GSH-Px), and (D) glutathione transferase (GST), and production of (E) ROS and (F) MDA in oxidative-damaged and control BMEC. Cells were treated with $\mathrm{H}_{2} \mathrm{O}_{2}(600 \mu M)$ for 6 h followed by transfection with scrambled small interfering RNA (siRNA) and NFE2L2 siRNA3 for $48 \mathrm{~h} .{ }^{*} P<0.05,{ }^{* *} P<0.01,{ }^{* * *} P<0.001$ compared with the control group. Error bars indicate SEM. 

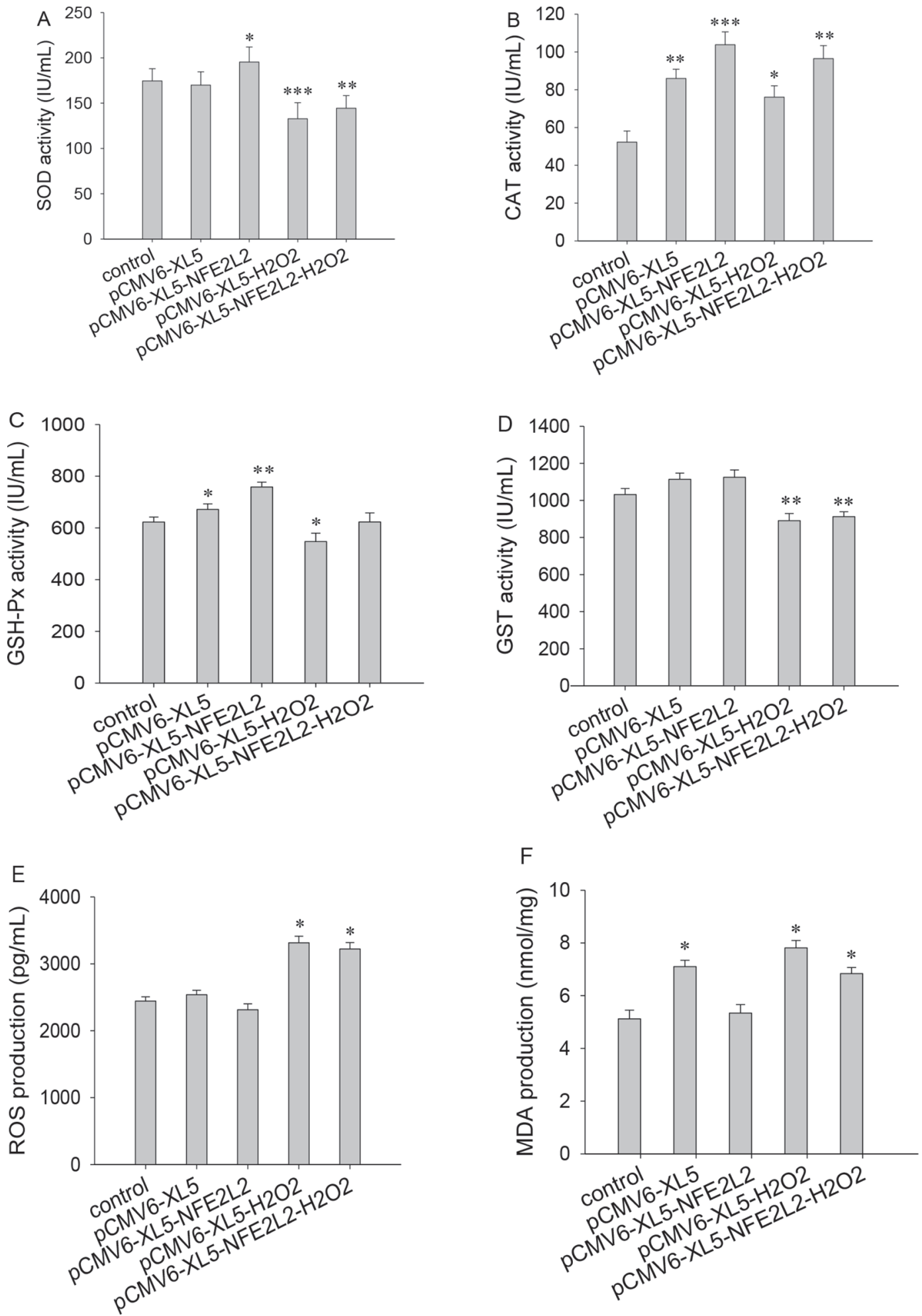

Figure 5. Effect of upregulating nuclear factor erythroid 2-related factor 2 (NFE2L2) expression on antioxidative enzyme activity, reactive oxygen species (ROS), and malondialdehyde (MDA) production in bovine mammary epithelial cells (BMEC). Activity of (A) superoxide dismutase (SOD), (B) catalase (CAT), (C) glutathione peroxidase (GSH-Px), and (D) glutathione transferase (GST) and production of (E) ROS and (F) MDA in oxidative-damaged and control BMEC. Cells were treated with $\mathrm{H}_{2} \mathrm{O}_{2}(600 \mu M)$ for 6 h followed by transfection with PCMV6XL5 NFE2L2 and pCMV6-XL5 for $48 \mathrm{~h} .{ }^{*} P<0.05,{ }^{* *} P<0.01,{ }^{* * *} P<0.001$ compared with the control group. Error bars indicate SEM. 
A
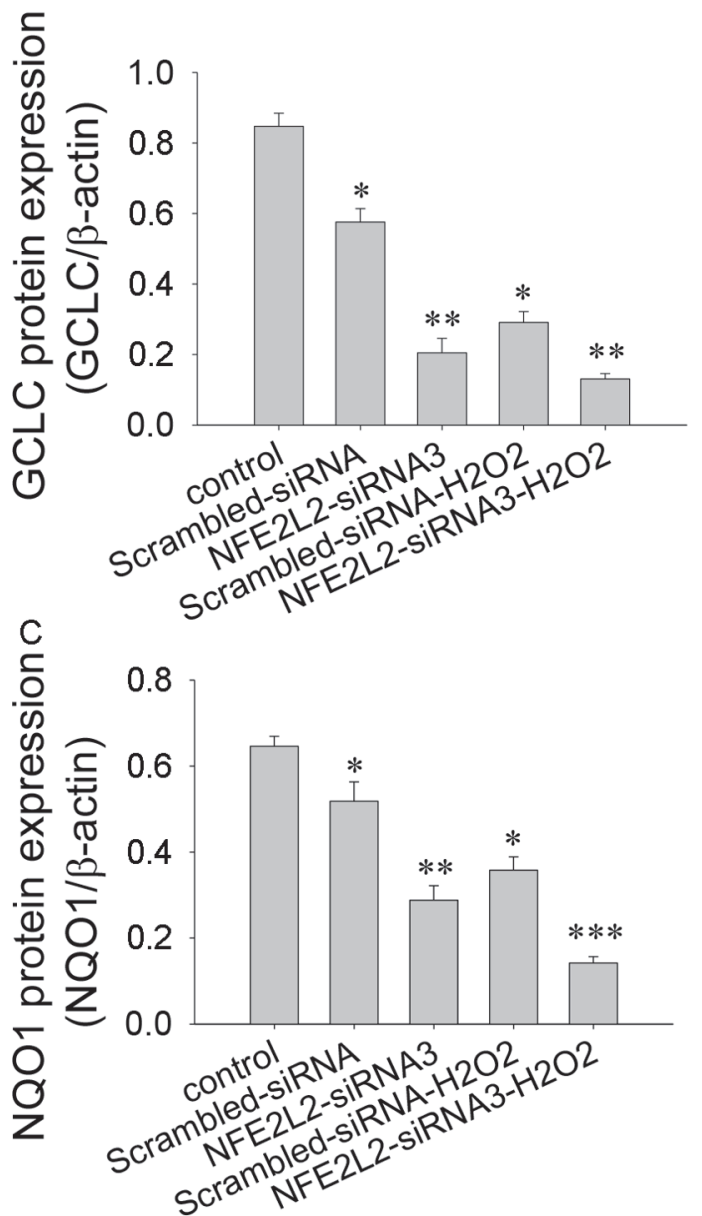

B

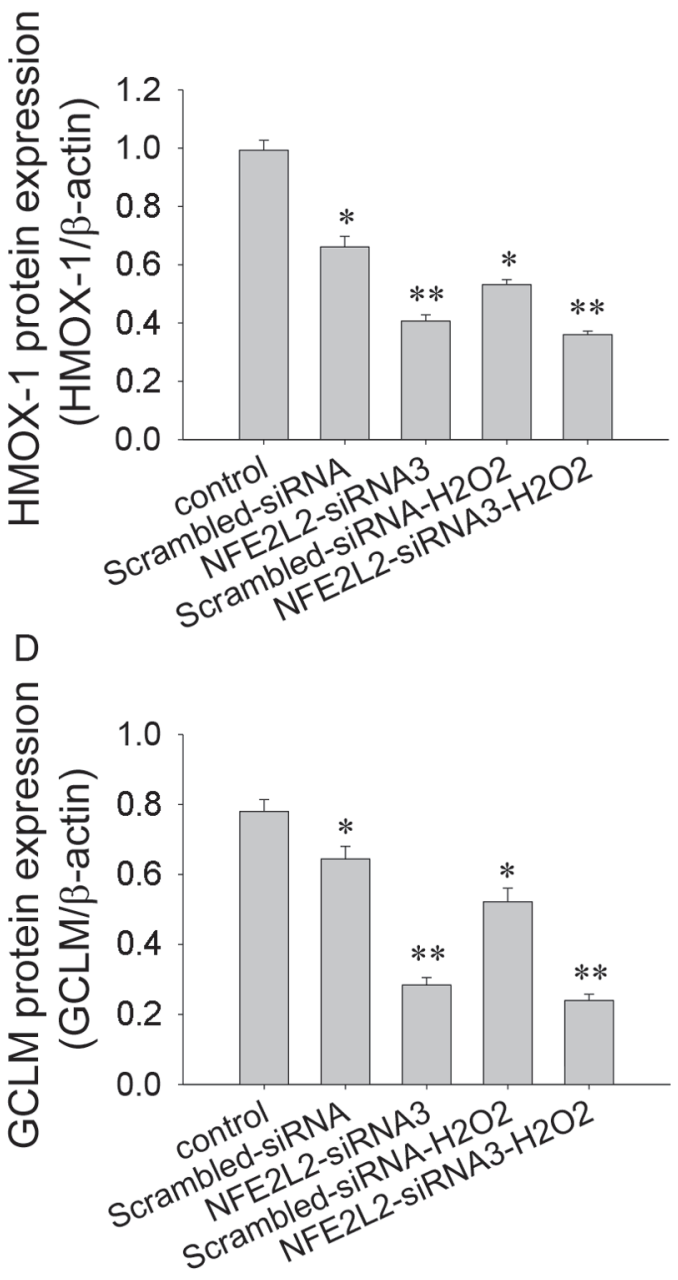

Figure 6. Effects of nuclear factor erythroid 2-related factor 2 (NFE2L2) small interfering RNA (siRNA)-3 interference fragment on downstream protein expression of NFE2L2 antioxidant response element signal pathway in bovine mammary epithelial cells (BMEC). Protein expression of (A) glutamate-cysteine ligase catalytic subunit (GCLC), (B) heme oxygenase-1 (HMOX-1), (C) NADH quinone oxidoreductase 1 (NQO1), and (D) glutamyl cystine ligase modulatory subunit (GCLM) was measured in oxidative-damaged and control BMEC by Western blot. Cells were treated with $\mathrm{H}_{2} \mathrm{O}_{2}(600 \mu M)$ for $6 \mathrm{~h}$ followed by transfection with scrambled small interfering RNA (siRNA) and NFE2L2 siRNA3 for 48 h. ${ }^{*} P<0.05,{ }^{*} P<0.01,{ }^{* * *} P<0.001$ compared with the control group. Error bars indicate SEM.

\section{DISCUSSION}

Taken together, the changes in BMEC proliferation rate, ROS and MDA production, and antioxidant enzyme activity in response to up or downregulation of NFE2L2 indicated that BMEC are susceptible to oxidative damage induced by $\mathrm{H}_{2} \mathrm{O}_{2}$. Thus, along with data from a previous study (Jin et al., 2016a), the data support the assertion that the NFE2L2-ARE signaling pathway is one of the main defense mechanisms of resistance against OS. Oxidative stress induced by $\mathrm{H}_{2} \mathrm{O}_{2}$ can increase ROS and MDA production, leading to decreased antioxidant ability, partly due to lower
NFE2L2 and HMOX-1 abundance. These responses in vitro could have bearing in vivo, particularly during times when the mammary gland experiences changes in metabolism or the number of potential challenges to normal function (e.g., mastitis).

Recent evidence, including our findings, indicated that dairy cows during the perinatal and transition into lactation periods are highly susceptible to altered redox (oxidation reduction) balance, resulting in OS (Abuelo et al., 2015; Hanschke et al., 2016). A large portion of this response is due to high metabolic rates and physiological adaptations to the onset of lactation. The period of negative energy balance during calving 
A

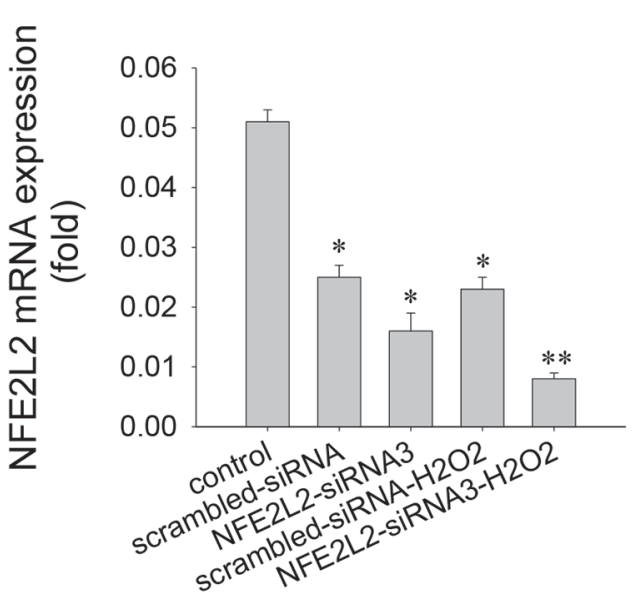

C
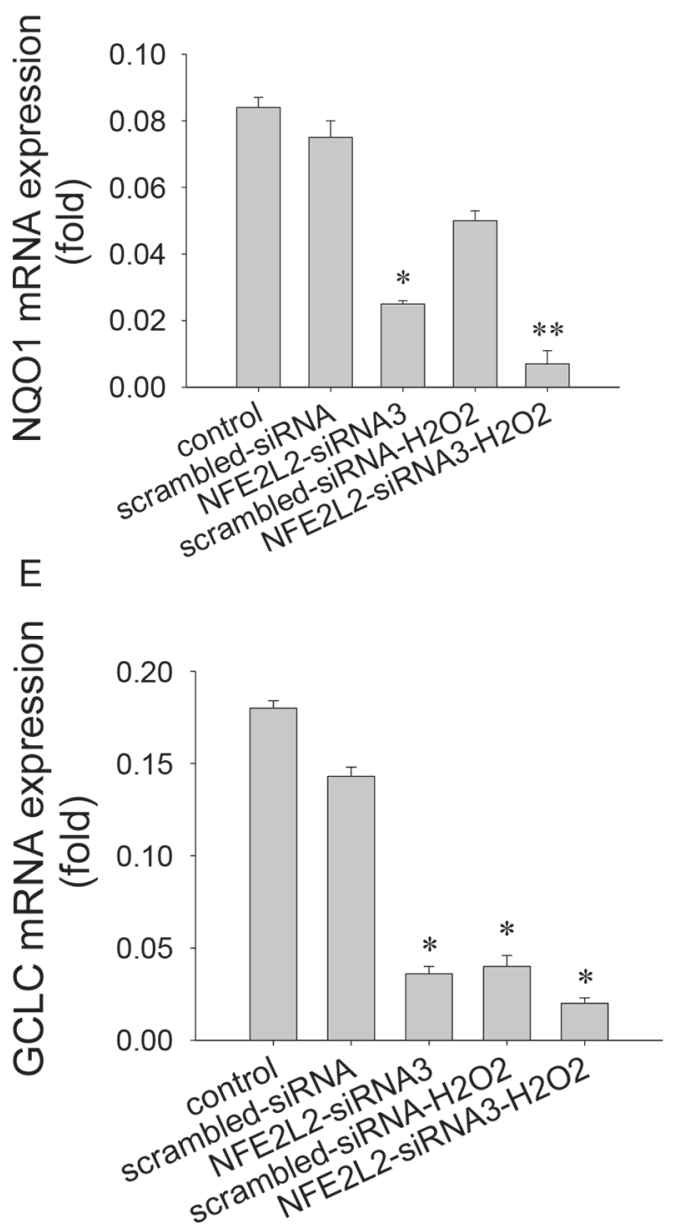

B

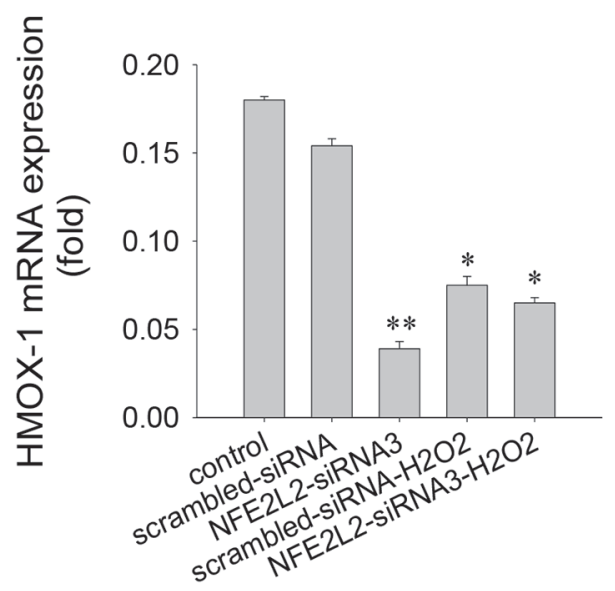

D

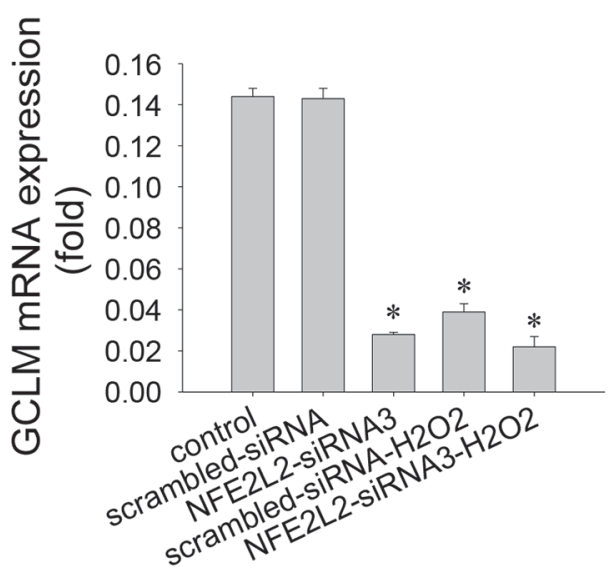

Figure 7. Effects of nuclear factor erythroid 2-related factor 2 (NFE2L2) small interfering RNA (siRNA)-3 interference fragment on downstream expression of target genes in the NFE2L2 antioxidant response element signaling pathway in bovine mammary epithelial cells (BMEC). The mRNA expression of (A) NFE2L2, (B) heme oxygenase-1 (HMOX-1), (C) NADH quinone oxidoreductase 1 (NQO1), (D) glutamyl cystine ligase modulatory subunit ( GCLM), and (E) glutamate-cysteine ligase catalytic subunit $(G C L C)$ was measured in oxidative-damaged and control BMEC by reverse-transcription PCR. Cells were treated with $\mathrm{H}_{2} \mathrm{O}_{2}(600 \mu M)$ for $6 \mathrm{~h}$ followed by transfection with scrambled small interfering RNA (siRNA) or NFE2L2 siRNA3 for $48 \mathrm{~h} .{ }^{*} P<0.05,{ }^{*} P<0.01$ compared with the control group. Error bars indicate SEM. 

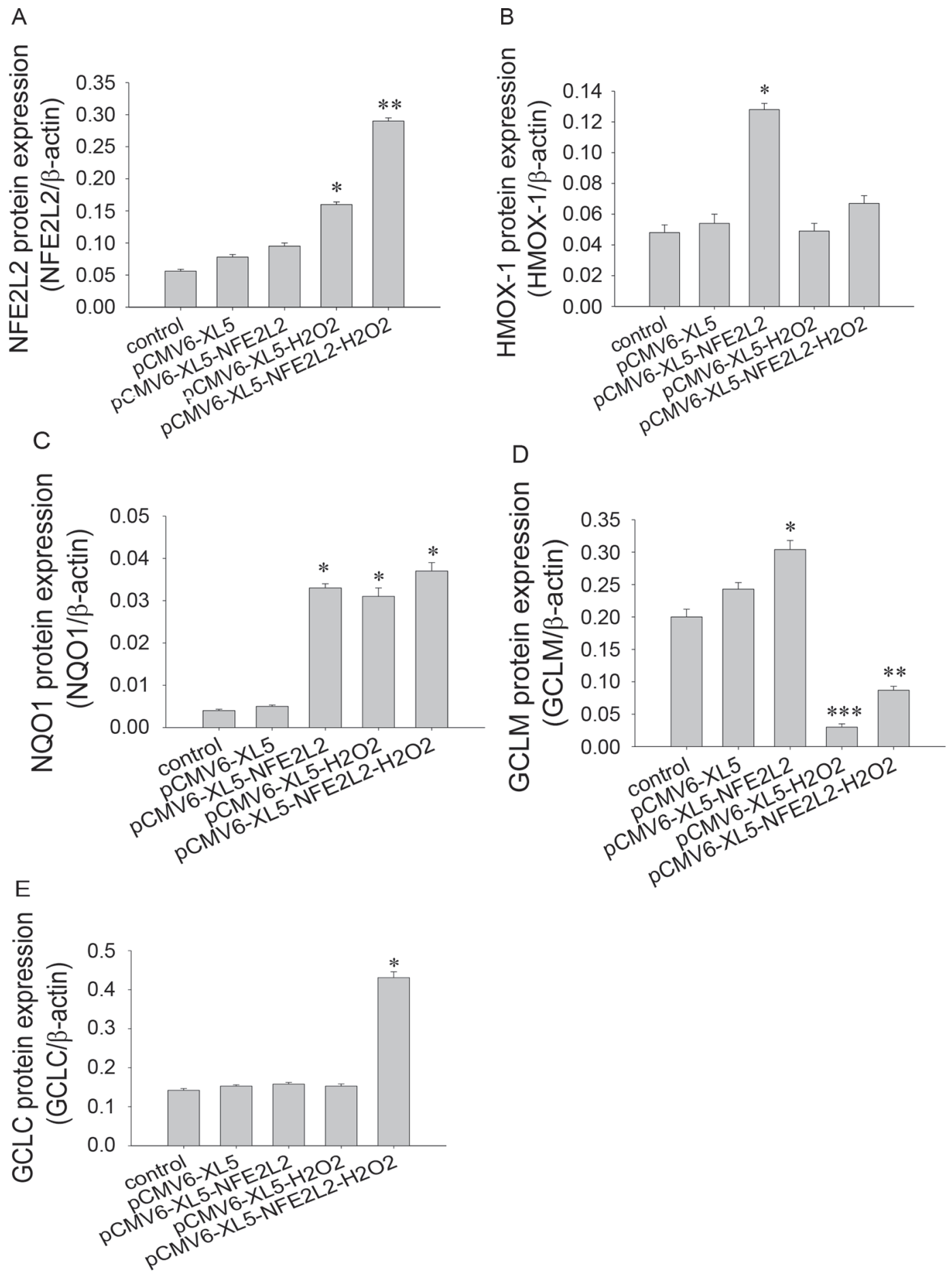

Figure 8. Effects of pCMV6-XL5 nuclear factor erythroid 2-related factor 2 (NFE2L2) overexpression vector on downstream protein expression of NFE2L2 antioxidant response element signaling pathway in bovine mammary epithelial cells (BMEC). Protein expression of (A) NFE2L2, (B) heme oxygenase-1 (HMOX-1), (C) NADH quinone oxidoreductase 1 (NQO1), (D) glutamyl cystine ligase modulatory subunit (GCLM), and (E) glutamate-cysteine ligase catalytic subunit (GCLC) in oxidative-damaged and control BMEC was measured by Western blot. Cells were treated with $\mathrm{H}_{2} \mathrm{O}_{2}(600 \mu M)$ for $6 \mathrm{~h}$ followed by transfection with pCMV6-XL5 and PCMV6-XL5 NFE2L2 for 48 h. ${ }^{*} P<0.05,{ }^{*} P$ $<0.01,{ }^{* * *} P<0.001$ compared with the control group. Error bars indicate SEM. 

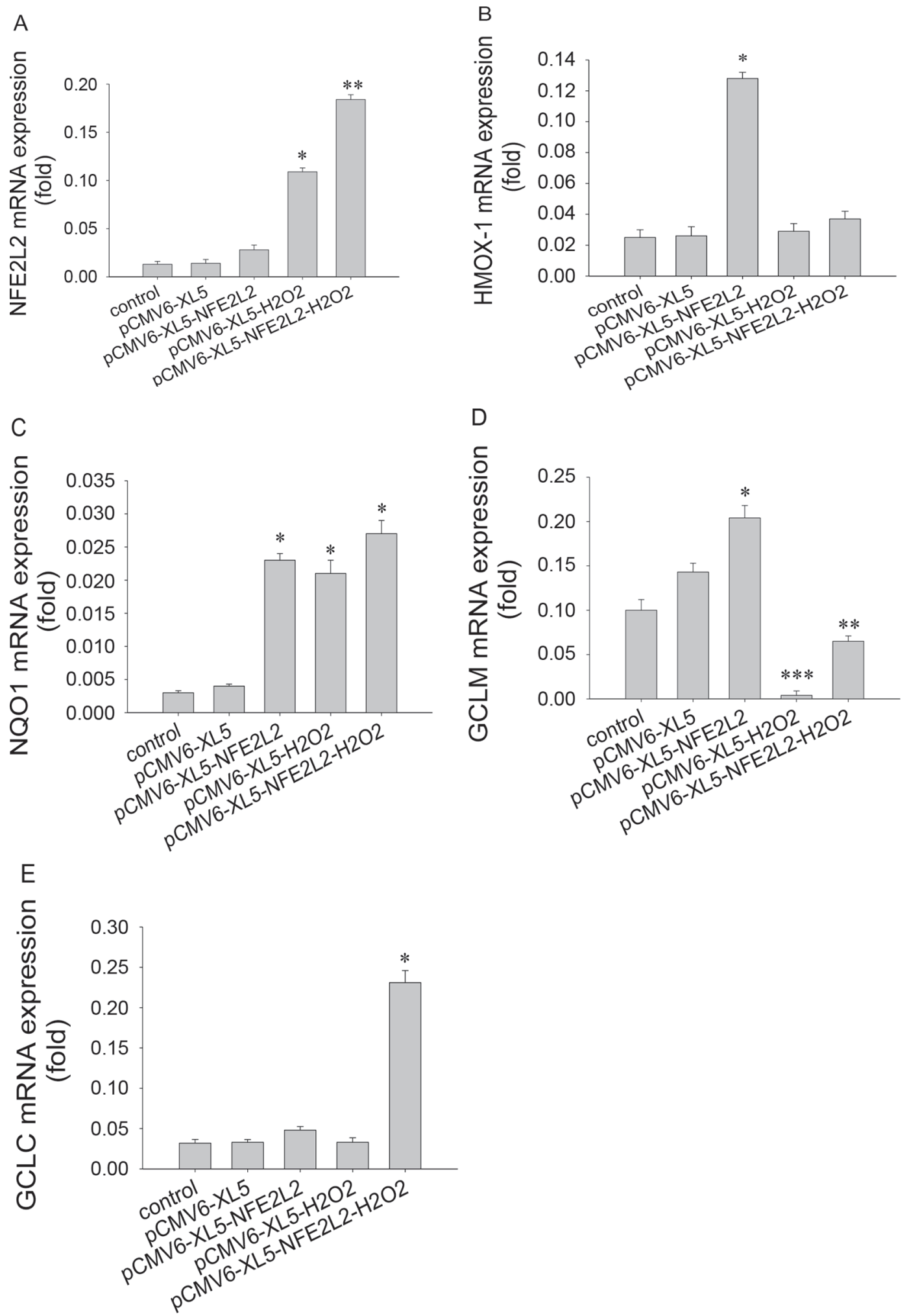

Figure 9. Effects of pCMV6-XL5 nuclear factor erythroid 2-related factor 2 (NFE2L2) overexpression vector on expression of target genes in the NFE2L2 antioxidant response element signaling pathway in bovine mammary epithelial cells (BMEC). The mRNA expression of (A) NFE2L2, (B) heme oxygenase-1 (HMOX-1), (C) NADH quinone oxidoreductase 1 (NQO1), (D) glutamyl cystine ligase modulatory subunit $(G C L M)$, and $(\mathrm{E})$ glutamate-cysteine ligase catalytic subunit $(G C L C)$ in oxidative-damaged and control BMEC was measured by reversetranscription PCR. Cells were treated with $\mathrm{H}_{2} \mathrm{O}_{2}(600 \mu M)$ for $6 \mathrm{~h}$ followed by transfection with pCMV6-XL5 and PCMV6-XL5 NFE2L2 for 48 h. ${ }^{*} P<0.05,{ }^{* *} P<0.01,{ }^{* * *} P<0.001$ compared with the control group. Error bars indicate SEM. 


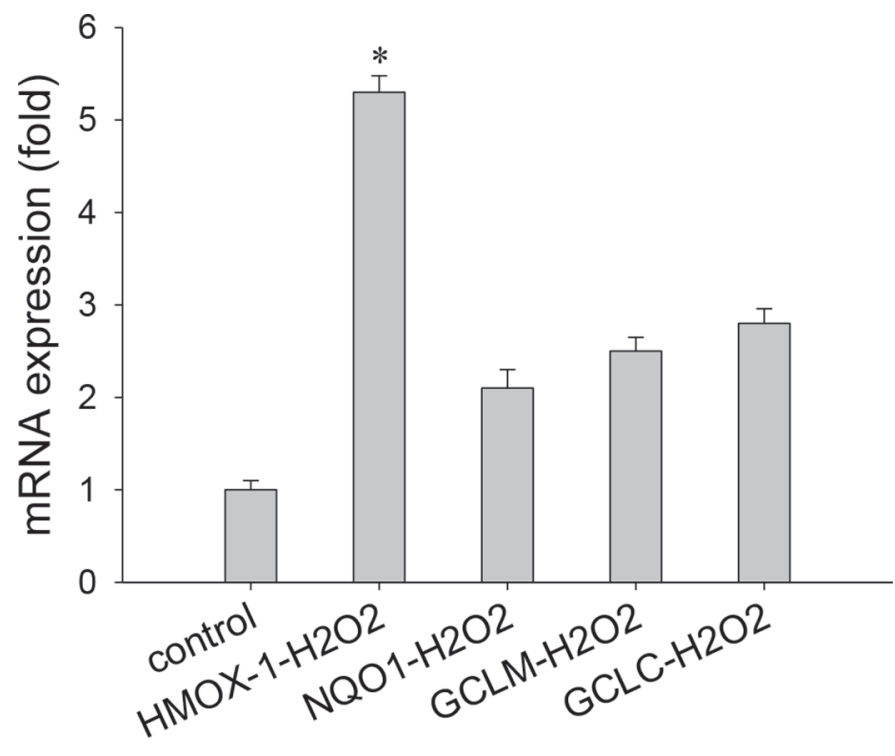

Figure 10. Downstream expression of gene targets in the nuclear factor erythroid 2-related factor 2 (NFE2L2) antioxidant response element pathway in bovine mammary epithelial cells under oxidative stress. The mRNA expression of heme oxygenase-1 (HMOX-1), NADH quinone oxidoreductase 1 (NQO1), glutamyl cystine ligase modulatory subunit $(G C L M)$, and glutamate-cysteine ligase catalytic subunit $(G C L C)$ was measured by reverse-transcription PCR after treatment of cells with $\mathrm{H}_{2} \mathrm{O}_{2}(600 \mu M)$ for $6 \mathrm{~h}$. ${ }^{*} P<0.05$ compared with the control group. Error bars indicate SEM.

and early lactation also can further cause disorders associated with OS (Bernabucci et al., 2005; Castillo et al., 2005; Patton et al., 2006; van Knegsel et al., 2007).

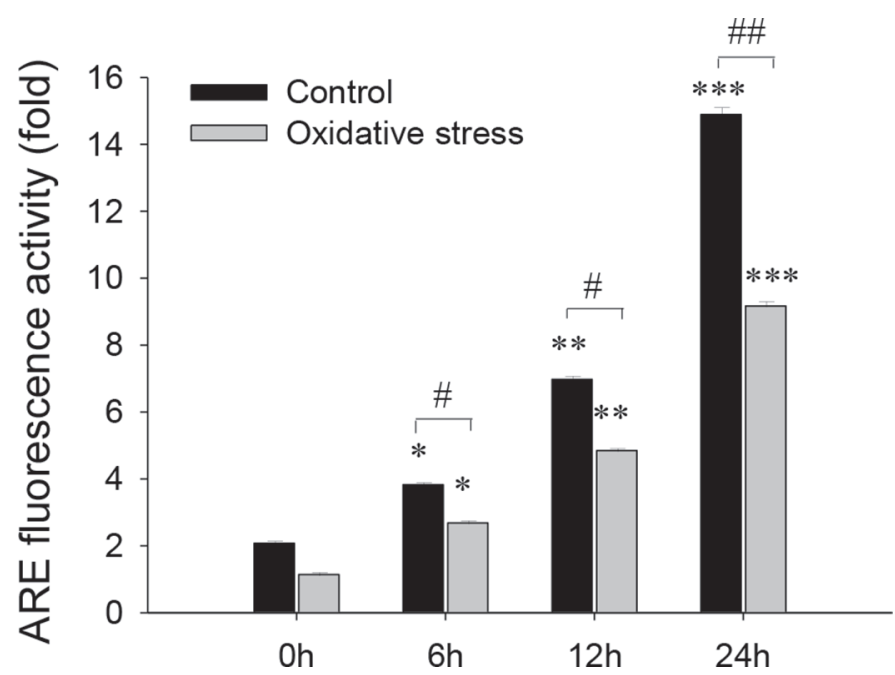

Figure 11. Fluorescence activity of antioxidant response element (ARE) in control and oxidative stress groups. The fluorescence activity of ARE was detected with the biluciferase gene reporter assay (E1910; Promega, Madison, WI) after treatment of cells with $\mathrm{H}_{2} \mathrm{O}_{2}$ $(600 \mu M)$ for $0,6,12$, and $24 \mathrm{~h}$. \#P<0.05, \#\#P<0.01 compared with the control group; ${ }^{*} P<0.05$, ${ }^{*} * P<0.01$, *** $P<0.001 \mathrm{com}-$ pared with $0 \mathrm{~h}$. Error bars indicate SEM.
An imbalance between ROS production and antioxidant defenses can alter normal cell function and decrease immune function in dairy cows (Sordillo et al., 2009). Thus, maintaining intracellular redox balance to decrease the damage induced by OS is important. Excess production of ROS and MDA or insufficient antioxidant enzymes may cause oxidative damage in BMEC that could compromise cell viability. The focus of the present study was on enzymatic antioxidants, and future research would have to assess the role of other molecules with antioxidative properties that are not enzymes.

In the present study, the lower activity of SOD, GSHPx, CAT, and GSH coupled with the higher content of ROS and MDA induced by an acute supraphysiologic concentration of $\mathrm{H}_{2} \mathrm{O}_{2}$ overpowered the ability of BMEC to scavenge ROS to prevent cell damage. The data underscore that these antioxidant systems were insufficient to counteract the increase in ROS and MDA in response to $\mathrm{H}_{2} \mathrm{O}_{2}$, causing cellular apoptosis and necrosis also. Whether such an acute oxidative attack mirrors the in vivo condition that periparturient cows may experience is unknown. Thus, results from the present study should be carefully translated to the in vivo condition. It is more likely that at the onset of lactation cows are exposed to a chronic increase in both ROS and reactive nitrogen species; hence, in vivo studies focusing on the NFE2L2-ARE signaling pathway should be performed to better address the relevance of in vitro findings from the present study.

At least in ruminant cells, NFE2L2 is one of the most important transcription factors regulating expression of several antioxidant or detoxifying enzymes through its interaction with ARE (Jin et al., 2016a). Recent studies have demonstrated a crucial function for the NFE2L2-ARE signaling pathway in regulating BMEC homeostasis under OS (Jin et al., 2016a,b). In fact, this pathway is an early sensor of OS, acting to dampen oxidative damage by upregulating the expression of phase II detoxifying enzymes (Lee and Johnson, 2004). Thus, the finding that up- or downregulating the expression of NFE2L2 could alter the degree of apoptosis induced by $\mathrm{H}_{2} \mathrm{O}_{2}$ underscores its importance for BMEC. Hence, we speculate that the vulnerability to oxidative damage and death in the mammary gland might depend heavily on NFE2L2.

In this study, we demonstrated that HMOX-1 is a key antioxidant gene that helps protect BMEC against OS, and it is under the control of NFE2L2 through the ARE (Bae et al., 2017). Previous studies confirmed that the expression of HMOX-1 is mediated by the ARE and depends on NFE2L2 activation (Jian et al., 2011). Thus, the present data confirm that NFE2L2 itself does not elicit an antioxidant effect but rather helps 
launch a series of transcriptional changes upregulating the expression of antioxidant detoxifying enzymes. The specific mechanisms whereby activated NFE2L2 travels to the nucleus and activates the transcriptional program remain to be elucidated.

Regardless of addition of $\mathrm{H}_{2} \mathrm{O}_{2}$, the dual-luciferase data revealing that treatment with $\mathrm{H}_{2} \mathrm{O}_{2}$ decreased the fluorescence intensity of ARE in a time-dependent manner along with the decrease in protein and mRNA expression of NFE2L2 under oxidative-damaged conditions suggested the existence of an "activation barrier" preventing NFE2L2-ARE signaling under oxidative damage conditions. Such an activation barrier of NFE2L2 can further aggravate the toxicity and damage of cells, resulting in physiological dysfunction leading to apoptosis or necrosis.

\section{CONCLUSIONS}

Our study provides evidence that OS can increase the oxidative damage of BMEC by reducing nuclear translocation and transcriptional activity of NFE2L2. As a result, several molecular and antioxidant enzymatic mechanisms are impaired, leading to an unbalanced redox state.

\section{ACKNOWLEDGMENTS}

This research was supported by grants from China National Natural Science Foundation of China (no. 31460616 and no. 31601975, Beijing) and from the China Agriculture Research System (no. CARS-36, Beijing). Y. Ma received a China Scholarship Council (Beijing) postdoctoral fellowship to train at the University of Illinois (Urbana).

\section{REFERENCES}

Abuelo, A., J. Hernandez, J. L. Benedito, and C. Castillo. 2015. A pilot study to compare oxidative status between organically and conventionally managed dairy cattle during the transition period. Reprod. Domest. Anim. 50:538-544. https://doi.org/10.1111/rda .12519 .

Ahn, J. Y., N. Aoki, T. Adachi, Y. Mizuno, R. Nakamura, and T. Matsuda. 1995. Isolation and culture of bovine mammary epithelial cells and establishment of gene transfection conditions in the cells. Biosci. Biotechnol. Biochem. 59:59-64. https://doi.org/10 $.1271 /$ bbb.59.59.

Bae, W. J., G. Q. Zhu, S. W. Choi, H. C. Jeong, F. Bashraheel, K. S. Kim, S. J. Kim, H. J. Cho, U. S. Ha, S. H. Hong, J. Y. Lee, H. A. Oh, H. C. Koo, D. R. Kim, S. Y. Hwang, and S. W. Kim. 2017. Antioxidant and antifibrotic effect of a herbal formulation in vitro and in the experimental andropause via Nrf2/HO-1 signaling pathway. Oxid. Med. Cell. Longev. 2017:6024839. https://doi.org/ 10.1155/2017/6024839.

Bernabucci, U., B. Ronchi, N. Lacetera, and A. Nardone. 2005. Influence of body condition score on relationships between metabolic status and oxidative stress in periparturient dairy cows. J. Dairy Sci. 88:2017-2026.
Bouwstra, R. J., M. Nielen, J. R. Newbold, E. H. Jansen, H. F. Jelinek, and T. van Werven. 2010. Vitamin E supplementation during the dry period in dairy cattle. Part II: Oxidative stress following vitamin E supplementation may increase clinical mastitis incidence postpartum. J. Dairy Sci. 93:5696-5706. https://doi.org/10.3168/ jds.2010-3161.

Castillo, C., J. Hernandez, A. Bravo, M. Lopez-Alonso, V. Pereira, and J. L. Benedito. 2005. Oxidative status during late pregnancy and early lactation in dairy cows. Vet. J. 169:286-292. https://doi.org/ 10.1016/j.tvjl.2004.02.001.

Deng, S., K. Yu, W. Jiang, Y. Li, S. Wang, Z. Deng, Y. Yao, B. Zhang, G. Liu, Y. Liu, and Z. Lian. 2017. Erratum to: Over-expression of Toll-like receptor 2 up-regulates heme oxygenase-1 expression and decreases oxidative injury in dairy goats. J. Anim. Sci. Biotechnol. 8:19. https://doi.org/10.1186/s40104-017-0149-5.

Fernández-Checa, J. C. 2003. Redox regulation and signaling lipids in mitochondrial apoptosis. Biochem. Biophys. Res. Commun. 304:471-479. https://doi.org/10.1016/s0006-291x(03)00619-3.

Goff, J. P. 2006. Major advances in our understanding of nutritional influences on bovine health. J. Dairy Sci. 89:1292-1301.

Hanschke, N., M. Kankofer, L. Ruda, M. Holtershinken, U. Meyer, J. Frank, S. Danicke, and J. Rehage. 2016. The effect of conjugated linoleic acid supplements on oxidative and antioxidative status of dairy cows. J. Dairy Sci. 99:8090-8102. https://doi.org/10.3168/ jds.2015-10685.

Howlett, A. R., and M. Bissell. 1993. The influence of tissue microenvironment (stroma and extracellular matrix) on the development and function of mammary epithelium. Epithelial Cell Biol. 2:79-89.

Jain, A. K., and A. K. Jaiswal. 2007. GSK-3beta acts upstream of Fyn kinase in regulation of nuclear export and degradation of NF-E2 related factor 2. J. Biol. Chem. 282:16502-16510. https://doi.org/ 10.1074/jbc.M611336200.

Jian, Z., K. Li, L. Liu, Y. Zhang, Z. Zhou, C. Li, and T. Gao. 2011. Heme oxygenase- 1 protects human melanocytes from $\mathrm{H}_{2} \mathrm{O}_{2}$-induced oxidative stress via the Nrf2-ARE pathway. J. Invest. Dermatol. 131:1420-1427. https://doi.org/10.1038/jid.2011.56.

Jin, X., K. Wang, H. Liu, F. Hu, F. Zhao, and J. Liu. 2016a. Protection of bovine mammary epithelial cells from hydrogen peroxideinduced oxidative cell damage by resveratrol. Oxid. Med. Cell. Longev. 2016:2572175. https://doi.org/10.1155/2016/2572175.

Jin, X. L., K. Wang, L. Liu, H. Y. Liu, F. Q. Zhao, and J. X. Liu. 2016b. Nuclear factor-like factor 2-antioxidant response element signaling activation by tert-butylhydroquinone attenuates acute heat stress in bovine mammary epithelial cells. J. Dairy Sci. 99:9094-9103. https://doi.org/10.3168/jds.2016-11031.

Kaspar, J. W., S. K. Niture, and A. K. Jaiswal. 2009. Nrf2:INrf2 (Keap1) signaling in oxidative stress. Free Radic. Biol. Med. 47:1304-1309. https://doi.org/10.1016/j.freeradbiomed.2009.07 .035 .

Kensler, T. W., N. Wakabayashi, and S. Biswal. 2007. Cell survival responses to environmental stresses via the Keap1-Nrf2-ARE pathway. Annu. Rev. Pharmacol. Toxicol. 47:89-116. https://doi.org/ 10.1146/annurev.pharmtox.46.120604.141046.

Kim, K. C., K. A. Kang, R. Zhang, M. J. Piao, G. Y. Kim, M. Y. Kang, S. J. Lee, N. H. Lee, Y. J. Surh, and J. W. Hyun. 2010. Upregulation of Nrf2-mediated heme oxygenase-1 expression by eckol, a phlorotannin compound, through activation of Erk and PI3K/ Akt. Int. J. Biochem. Cell Biol. 42:297-305. https://doi.org/10 .1016/j.biocel.2009.11.009.

Kruk, J., and E. Duchnik. 2014. Oxidative stress and skin diseases: Possible role of physical activity. Asian Pac. J. Cancer Prev. 15:561-568. https://doi.org/10.7314/apjcp.2014.15.2.561.

Lee, J. M., and J. A. Johnson. 2004. An important role of Nrf2-ARE pathway in the cellular defense mechanism. J. Biochem. Mol. Biol. $37: 139-143$

Li, H., F. Song, L. R. Duan, J. J. Sheng, Y. H. Xie, Q. Yang, Y. Chen, Q. Q. Dong, B. L. Zhang, and S. W. Wang. 2016. Paeonol and danshensu combination attenuates apoptosis in myocardial infarcted rats by inhibiting oxidative stress: Roles of Nrf2/HO-1 and 
PI3K/Akt pathway. Sci. Rep. 6:23693. https://doi.org/10.1038/ srep23693.

Loor, J. J., M. Bionaz, and J. K. Drackley. 2013. Systems physiology in dairy cattle: Nutritional genomics and beyond. Annu. Rev. Anim. Biosci. 1:365-392. https://doi.org/10.1146/annurev-animal $-031412-103728$.

Miranda, S. G., N. G. Purdie, V. R. Osborne, B. L. Coomber, and J. P. Cant. 2011. Selenomethionine increases proliferation and reduces apoptosis in bovine mammary epithelial cells under oxidative stress. J. Dairy Sci. 94:165-173. https://doi.org/10.3168/jds .2010-3366.

Nguyen, T., P. J. Sherratt, and C. B. Pickett. 2003. Regulatory mechanisms controlling gene expression mediated by the antioxidant response element. Annu. Rev. Pharmacol. Toxicol. 43:233-260. https://doi.org/10.1146/annurev.pharmtox.43.100901.140229.

Osorio, J. S., P. Ji, J. K. Drackley, D. Luchini, and J. J. Loor. 2014 Smartamine $\mathrm{M}$ and MetaSmart supplementation during the peripartal period alter hepatic expression of gene networks in 1-carbon metabolism, inflammation, oxidative stress, and the growth hormone-insulin-like growth factor 1 axis pathways. J. Dairy Sci. 97:7451-7464. https://doi.org/10.3168/jds.2014-8680.

Patton, J., D. A. Kenny, J. F. Mee, F. P. O'Mara, D. C. Wathes, M. Cook, and J. J. Murphy. 2006. Effect of milking frequency and diet on milk production, energy balance, and reproduction in dairy cows. J. Dairy Sci. 89:1478-1487. https://doi.org/10.3168/ jds.S0022-0302(06)72215-9.
Sordillo, L. M., and S. L. Aitken. 2009. Impact of oxidative stress on the health and immune function of dairy cattle. Vet. Immunol. Immunopathol. 128:104-109. https://doi.org/10.1016/j.vetimm.2008 .10 .305 .

Sordillo, L. M., G. A. Contreras, and S. L. Aitken. 2009. Metabolic factors affecting the inflammatory response of periparturient dairy cows. Anim. Health Res. Rev. 10:53-63. https://doi.org/10.1017/ S1466252309990016.

Szőcs, K. 2004. Endothelial dysfunction and reactive oxygen species production in ischemia reperfusion and nitrate tolerance. Gen. Physiol. Biophys. 23:265-295.

Talalay, P. 2000. Chemoprotection against cancer by induction of phase 2 enzymes. Biofactors 12:5-11.

van Knegsel, A. T., H. van den Brand, J. Dijkstra, W. M. van Straalen, M. J. Heetkamp, S. Tamminga, and B. Kemp. 2007. Dietary energy source in dairy cows in early lactation: Energy partitioning and milk composition. J. Dairy Sci. 90:1467-1476. https://doi.org/ 10.3168/jds.S0022-0302(07)71632-6.

Zarzyńska, J., B. Gajkowska, U. Wojewódzka, E. Dymnicki, and T. Motyl. 2007. Apoptosis and autophagy in involuting bovine mammary gland is accompanied by up-regulation of TGF-beta1 and suppression of somatotropic pathway. Pol. J. Vet. Sci. 10:1-9.

Zhang, H., A. M. Gomez, X. Wang, Y. Yan, M. Zheng, and H. Cheng. 2013. ROS regulation of microdomain $\mathrm{Ca}(2+)$ signalling at the dyads. Cardiovasc. Res. 98:248-258. https://doi.org/10.1093/cvr/ cvt050. 